\title{
DOCUMENTATION
}

\section{Exécution de la percée du lac d'Artouste en vue de son aménagement en réservoir saisonnier}

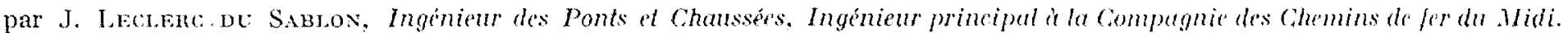

l.e lace d'Artouste, situé au plect du P'alas (2.976 m.), sur les confins des départements des Hautes-Pyrines et des BassesPyrénées, a pour émissaire le Sousoneou, affluent du Gave d'Ossau.

La situation géographique du lac d'Artouste, situé a une alt1tude élevée $(1.967 \mathrm{~m}$. 50 ) et alimenté par un bassin versant important $\left(7,45 \mathrm{~km}^{2}\right)$, en fait un réservoir naturel de premier ordre. dont l'utilisation comme régulateur annuel est à la base de l'aminagement hydro-électrique de la Vallée d'Ossau, entrepris par la Compagnie des Chemins de fer du Midi.

La capacité à donner au réserroir a été fixcée, à la suite d"une étude méthodique, des débits réalisés pendant une série d'années, à $23.000 .000 \mathrm{~m}^{3}$. L'étude topographique du lac a montré

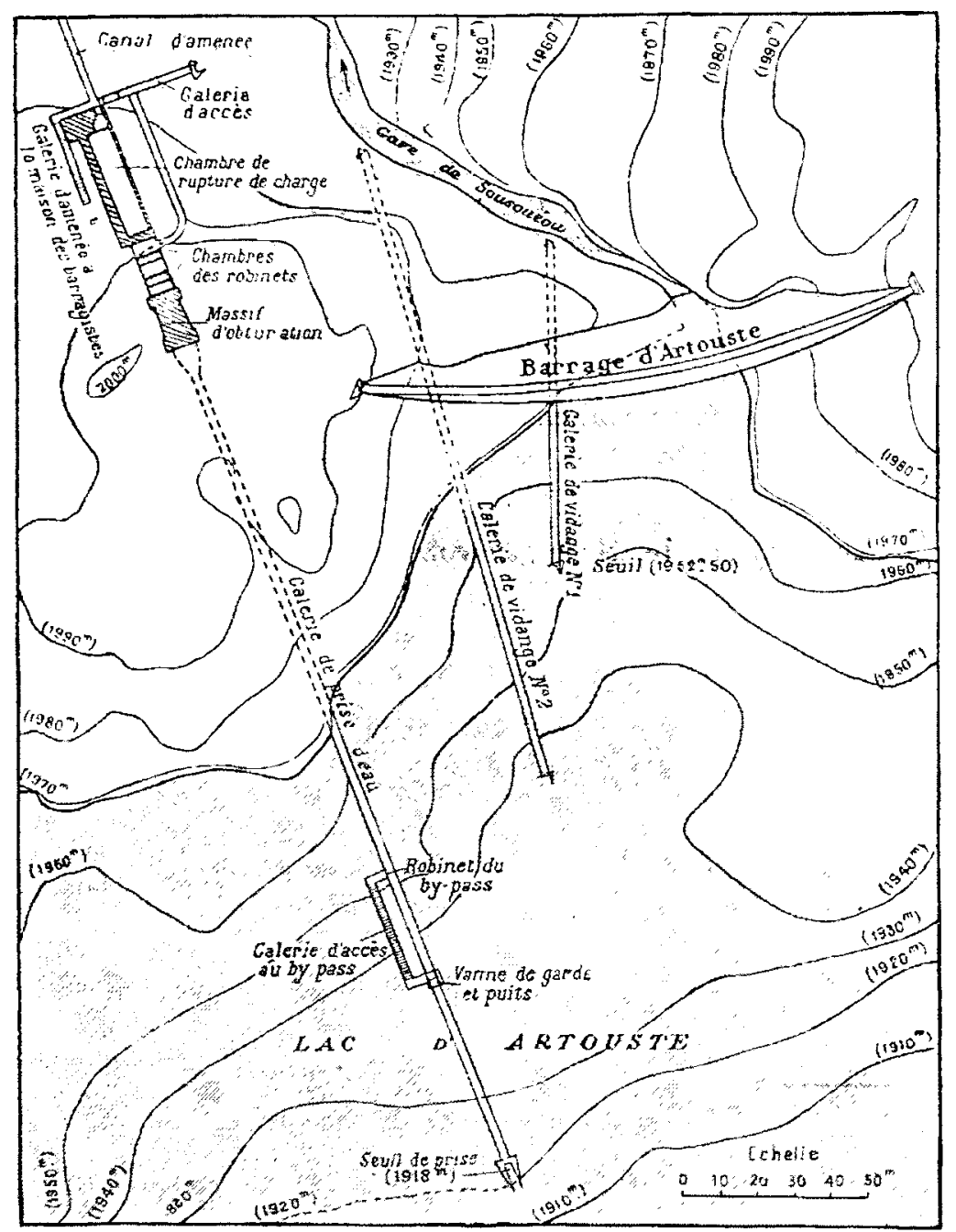

Frg. 1. - Plan d'ensemble de la Prise d'eau el des galeries de vidange du lac d'Arbouste - Echelle : 0,001 p. m.

que cette capacité pouvait être olotenue dans de bonnes conclitions en perçant le lac a $49 \mathrm{~m}$. 50 en dessous de son niveau normal, et en relevant ce niveau de 22 mètres au moyen d'un barrage.

La présente note a pour objet la description des travaux exécutés à l'occasion de la percée du lac.

\section{1}

Il ent the possible de pratiquer clirectement la percée a la cote voulue : etant donne li charge d'eau considerable correspondante et les diffieultes qui en pouvaient résulter, in ful dicioi de pratiquer deux galeries auxiliaires, de manière à ne pas clépanscr une charge de 20 metres sur l'orifice. Les emplacements di res galeries furent choisis en voo d'une longueur minimum. l.at fig. I (Plan en courbes) montre la disposition des lieux. I,es fig. 2 et 3 précisent les profils en travers suivant l'axe des deux galeries, d'après le plan en courbes du lac levé au cours des études préliminaires. La perforation fut exćcuté au moyen de perforatrices à colonne Ingersoll.

L'Avancement des galeries fut mene sur une section normale de $1 \mathrm{~m} .80$ de largeur sur $1 \mathrm{~m}$. 80 de hauteur environ. Pour obtenir cette section dans le sranit compact qui constitue la cuvette du lac d'Artouste, on dut pratiquer des volées de 13 a 1.1 trous le mine, profonds de $1 \mathrm{~m}$. 50 environ : + trous au centre de la galerie répartis sur une cironference de $0 \mathrm{~m}$. 60 sle diametre et cliriges en profondeur vers te centre de cette circonférence, constituant le foyer de la volée : les antres trous répartis sur le pour tour de la section. le diamctre des trous est de $10 \mathrm{~m}$ environ. La charge, constituce par des cartouches de dynanite gomms de $30 \mathrm{~m}$ de diametre, pesant 100 grammes chacune, est, apres achevement de la perforation, répartie dans les trous de mine. de façon a obtenir une charge totale de 7 a 10 kilos par volé suivant la dureté et la disposition des banes de rocher traversés. La mise à feu fut pratiqué par miche à mine ordinalre. Les coup. du foyer explosent d'abord le mamicre à former une excavation centrale sur laquelle les coups de pourtour font ahatage.

l a profondeur moyenne des avancements obtenus après chaque volie fut de $1 \mathrm{~m} .20$ a $1 \mathrm{~m}$. 40. Il fut possible, sauf dans les derniers metres, de pratiquer rigulierement 2 voles par jour

II

La galerie $n^{*} 1$ devait avoir 90 metres de longueur an maximum. La figure 2 montre les variations ohservées dans la nature et la tenue de la roche au cours de la perforation. Les parois. seches pendant les 40 premiers mètres (bon granit), présentèrent cnsuite ales suintements particls : l'humidite devint sensible i partir de 70 mètres : de fortes venues d'eau apparurent à 78 mètres. Le front de taille était precécie continuellement de sondages (en calotte en raison de la disposition de la plus courte distance probable à la paroi du lac); le 17 octobre 1924 , l'avancement atteignant $82 \mathrm{~m}$. 50 , ces sondases percèrent te fond du lac à environ $3 \mathrm{~m}$. 20 : par les trous correspondants jaillit de l'eau entrainant du sable et du gravier. The fois ces trous obturés, l'avancement fut continué par petites volćes jusqu'à $81 \mathrm{~m}$. 50, la roche étant toujours de bonne qualite. A ce moment on traversa une fissure avec venues d'eau abonlantes. l)e nouveaux sondages révélerent une épaisseur de paroi de 2 mètres : e'est alors que fut préparé le fourneau destiné à effectuer la percée (32 $\mathrm{kg}$.).

contrairement a l'attente, le jeu de ce foumeau (le 22 octobre a midi) ne donna aucun resultat.

Le débit n’ayant pas varié, ou presque, l'acees de la galeric ćtait toujours possible; il fut constaté que l'explosif avait jouc dans une poche de glaise qui en avait atténué l'effet.

Ine deuxième tentative, avec l'emploi d'une forte charge (225) kg. de dynamite), eut lieu le 23 octobre : le fourneau était placé en calotte dans me acvilé produjte par la précédente explosion. Un important bourrage constitué par un mur en sacs a terre était disposé derrière la chamse. L'allumage se fit par amorc' électrique dont la mise à feu s'obtient au. moyen d'une magnéto 
i main d'un lyje spicial. La percée lut réalisce, ainsi quen fit preuve la violence du phénomène cóté lac; un bloc de près de $1 \mathrm{~m}^{3}$ fut projeté et rléplacé, comme on put le constater par la suite, de plusieurs dizaines de mètres. Le débit de la galerie augmenta progressivement pour se fixer à $400 \mathrm{l} / \mathrm{s}$ environ au bout de 10 minutes. Ce débit etait manifestement insuffisant pour assurer la vidange du lac et permettre avant les fortes chutes de neige la mise de la galerie en ctat de débiter les hautes eaux du bassin versant au printemps suivant, condition indispensable à l'accomplissement du programme.

L'accès de la galerie étant encore possible, un examen détaillé en fut fait : elle fut trouvée obturéc par des ćboulis très abondants provenant, semblait-il, tant du lac, que et surtout, des parois qui se dislocpuaient, avec chute de blocs de la calotte sous l'action de chocs légers.

Dans ces conditions, on ne pouvait prudemment songer à attaquer ces ćboulis à la main : on procéda par explosions successives, avec de petites charges, car une forte charge aurait (le la température a cette altitude. Le travail fut repris le 21 juin 1925 et l'avancement continuait normalement, atteignant 172 mètres, le 7 juillet, quand l'examen du front d'attaque apres la volée du soir montra que la paroi du rocher en place avait été crevée, en calotte et à gauche, sur plus de $1 \mathrm{~m}^{2}$. L'éboulis, composé de galets arrondis et de sable tenait sans couler, le debit des infiltrations étant de l'ordre de $1 \mathrm{l} / \mathrm{s}$. Le lendemain matin, la situation paraissait inchangée et l'équipe de service se préparait à exécuter des boisages de consolidation : vers 11 heures, quelques cailloux tombèrent, et les venues d'eau se firent plus fortes : le chef mineur fit aussitôt évacuer la galerie : quelques minutes après, le débit augmenta pour atteindre rapidement $18 \mathrm{~m}^{3} / \mathrm{s}$ environ : la tranche d'eau comprise au-dessus de la galerie $n^{\circ} 2$ fut rapidement écoulée, l'abaissement du niveau atteignant :

$\begin{aligned} 5 \mathrm{~m} .50 & \text { en } 24 \mathrm{~h} . \\ 11 \mathrm{~m} . & \text { en } 48 \mathrm{~h} . \\ 14 \mathrm{~m} . & \text { en } 72 \mathrm{~h} .\end{aligned}$

$11 \mathrm{~m}$. en $72 \mathrm{~h}$.

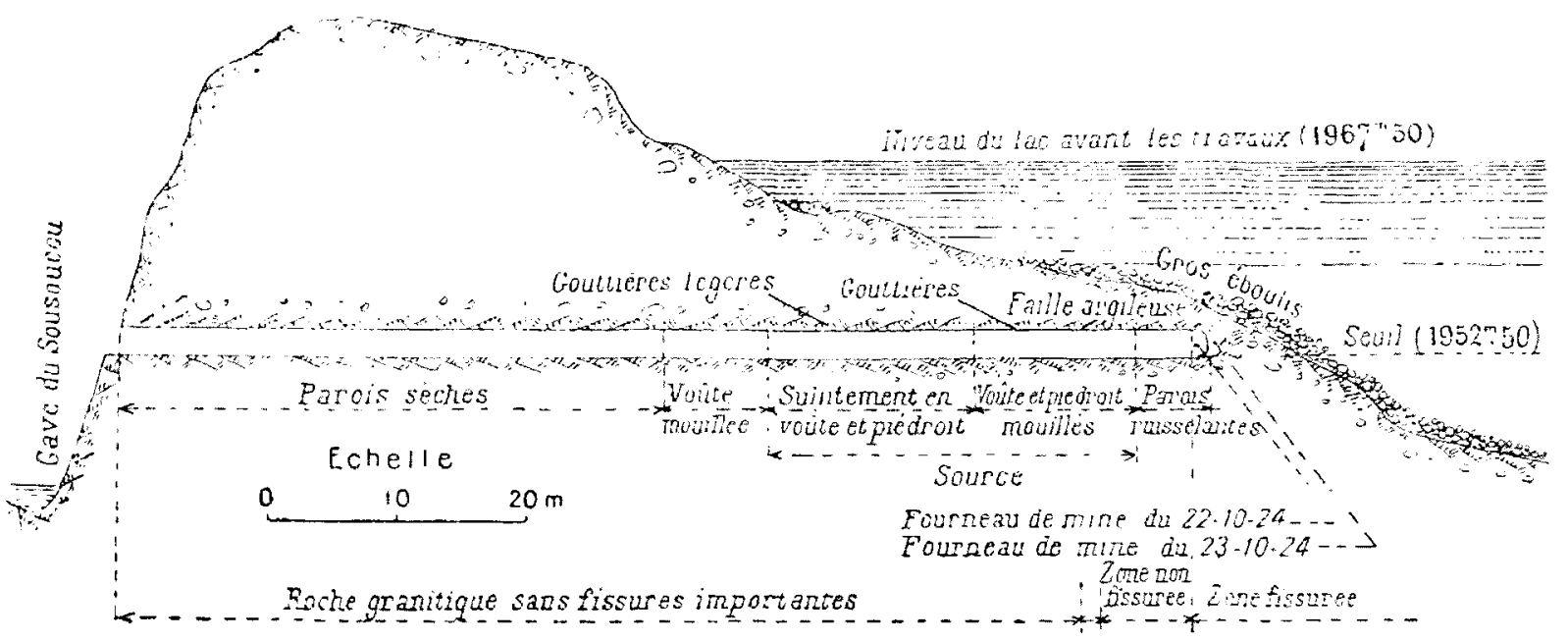

$\mathrm{Fig} 2$ - Galene $\mathrm{n}^{\mathrm{e}}$ 1 : Coupe donnant les venues deau et failles rencontrées a la perfoarton

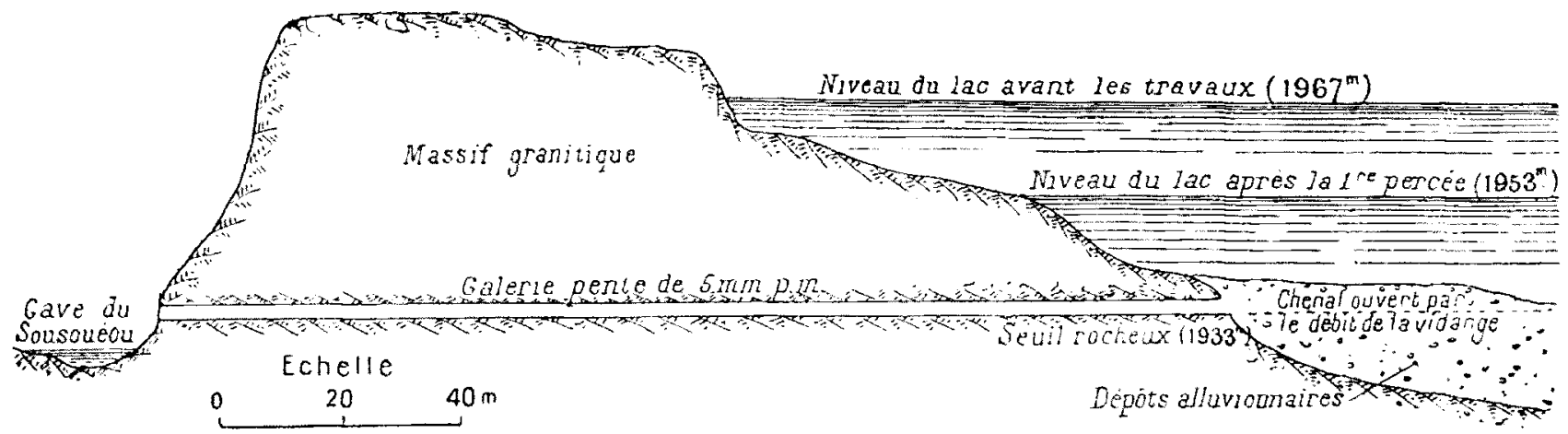

Fia. 3.- Conpe du terrain suivant laxe de la galerie $n^{\circ} 2$

pu causer un effondrement de la calotte. Deux explosions suffirent a porter le débit à 2.500 litres, chiffre largement suffisant pour le but exposé ci-dessus : aussi le travail fut-il arrêté le 27 octobre.

En raison du fait que les chutes de neige ont été presque nulles dans les Pyrences au cours de l'hiver 24-25, jusquen février, il fut possible de suivre de près l'état du lac : une reconnaissance exécutée all début de févier permit de constater que la partie supérieure de lorifice de vidange était hors de l'eau: le débit atteignait alors environ $600 \mathrm{l} / \mathrm{s}$. Une expédition fut organisée sur-le-champ et locquipe envoyée sur les lieux róussit à dégager entierement l'orifice. On constata que la galerie avait débouchó dans une zone d'ćboulis, ce qui explique les difficultés rencontrées.

Il est à noter (que ce travail n'a pu être exćcuté en temps vouhu que gràce à des circonstances atmosphériques exceptionnelles, l'accès du lac d'Artouste en période d'enneigement important relevant plutot des sports d'hiver que de la pratique des tra vaux.

\section{III}

La galerie $n^{\circ} 2$ ou galerie intermédiaire avait été poussée pendant l'été 1924; l'avancement atteignait $130 \mathrm{~m}, 20$ au $15 \mathrm{no}$ vembre, late où le chantier dut être levé en raison de la rigueur
Le 12 au soir, la vidange itait termince.

L'examen du lac montre que, dans la zone récemment évacuée par les eaux se trouvent des bancs considérables de vase et de glaise mouvante contenant de nombreux galets : il parait probable que les suintements ont progressivement délavé l'aggloméré de son liant naturel en criant des canalicules qui, une fois frayés, se sont rapidement élargis jusqu'à destruction totale du bouchon existant devant l'orifice.

Ce processus explique la durée relativement longue de ce débouchage naturel (1s heures environ).

Par la suite, l'érosion a creust aux abords de l'orifice un énorme cratère dans la masse des vases et dépòts glaiseux. Ces dépòts, fluides quand ils sont humides, deviennent durs en séchant.

Des phénomènes analogues furent constatés et continuent a se produire dans les zones traversées par les divers torrents, permanents ou saisonniers, qui alimentent le lac d'Artouste : là où la berge du lac est formée de graviers fins ou de sable, le ravinement a eu des effets importants, creusant des lits de plusieurs dizaines de mètres de profondeur, et déplaçant des dizaines de milliers de mètres cubes de déblais: lesdits déblais provenant d'ailleurs d'apports antírieur's des mêmes torrents, déversés dans les eaux calmes du lac. 
Lat troisieme gateric destince a servir par la subte de galeric de prise définitive a été perforée au cours de la campagne d'eté 1925 : elle est entièrement située dans le rocher en place, oì la vitesse d'avancement atteignit jusquà 3 mètres par jour. A cette galerie a ité adjoint un puits vertical dont la destination est la suivante (fig. f) : une vame de garde avec by-pass manouvre du sommet de ce puits permet d'obturer a volonte la galerie de prise quand le niveau de l'eau dans le lac est plus bas que l'orifice du puits : tone galerie oblique partant de cet orifice permet alors d'accéder a la partie amont de la robinetterie et de procider aux menues réparations, démontages, mise en place de bouchons, que certaines avaries peuvent rendre nécessaires. Sans ce dispositif, on doit, pour ces opérations, attendre la vidange complète du réservoir : et, même en étiage, les apports naturels gênent considérablement pour l'accès à la galerie de prise jusqu’à nécessiter l'emploi de scaphandres.

Ce puits, servant de cheminée pour l'aération et l'évacuation des déblais, facilita notablement l'achèvement de la galerie, qui* au total, a 300 mètres de long. D'importantes venues d'eau se faisaient jour dans le puits alors que la galerie est demeuréc jusqu'au bout parfaitement sèche.

Le 31 octobre, les sondages, pratiquées après chaque volee, perature ful tres incegale pendant linver 25-26, les chules ac neige alternant avec les périodles oin le vent chaud dit "vent d'Espagne "provoquait une abondante fonte de neige, et partant. une forte alimentation du lac.

Il fut décide dopérer comme l'amée précédente, èest-à-dire de procider à l'examen, et. le cais cichount, au degagement de lorifice còte lac pendant la saison iroitle, ou les apponts du bassin versant du lac sont très reduits: . un detachement d'ouvriers monta done au lac le 1 mars 1926. L 'omneigement etait tris favorable ì cette expédition : la neige étail très dure, le temps parfaitement clair et le personnel put passer sans aucume difficulte le col d'Arrius (2.25).

Le lat "Artouste itait couvert d'une couche ćpaisse de neige (1 $\mathrm{m}$. so en moyenne), la glace étail fondue au débouché du ruissean d'Arremoulit, émissaire des laes dlu même nom, et également à l'extrémité opposée du lac, vers le point où devait déboucher la galerie de percéc. On constata que le niveau de l'eau était à 8 mitres environ au-lessus de la cote qu'il aurait dù avoir si la perce avait eu lieu normalement, et que les caux s'ecoulaient dans une faille pratiquée entre le rocher on place et une grande dalle très cléclive dont l'extrémité superieure émergeait (v. fig. 5a); rocher et dalle ctaient dailleurs recouverts d'une couche epaisse

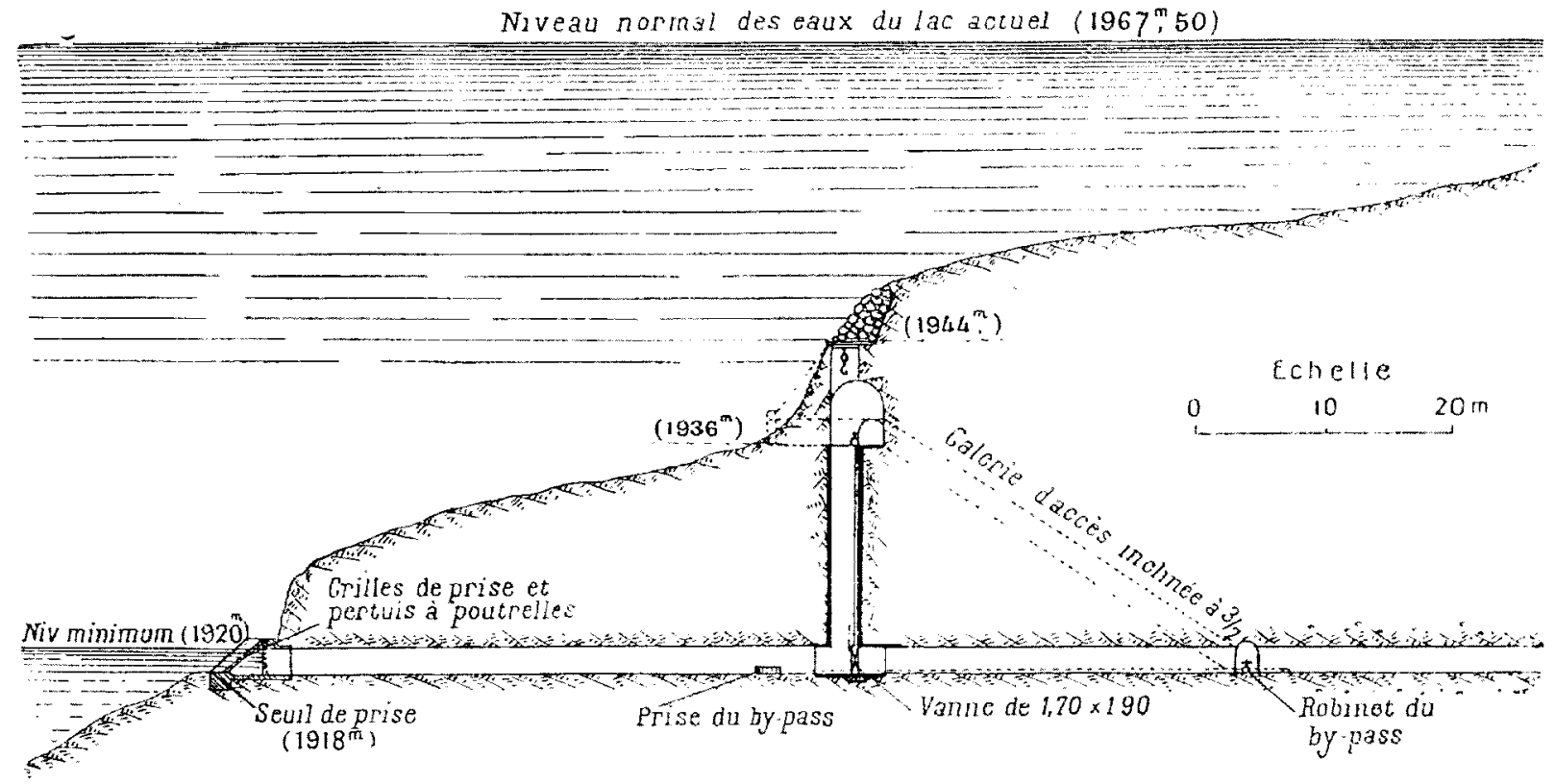

Fro. 1. - Tote de la prise d'eau : vame de garde, pults el palerie inclinéc d'acès au by-pass.

donnèrent des venues d'eau sous forte charge, et sans que la barre à mine éprouve de résistance, à 2 mètres environ du front de la galerie : on en conclut que ces sondages débouchaiènt dans le lac. On fit exploser, le 13 novembre 1925 , un fourneau de 275 kilos de dynamite soigneusement maintenu par un mur maçonné formant bourrage. Cette opération domna un débit d'abord faible, mais qui atteignit en quelques minutes la valeur de $1 \mathrm{~m}^{3} / \mathrm{se}$ conde. L'eau sortait très sale, ce qui indiquait le délavage d'une couche superficielle de vase, analogue à celle décrite plus haut.

En raison du faible tirant d'eau, il fut possible de remonter la galerie de prise, ce qui permit de constater la présence de déblais en abondance : l'explosion d'une faible charge d'explosifs disposée au fond de la galerie, détermina semble-t-il une dislocation du tampon formé par ces déblais et le débit passa de 1 à $2 \mathrm{~m}^{3} / \mathrm{s}$.

Il y a lieu de remarquer que les conditions d'exécution du travail devinrent à la fin très difficiles par suite d'une période de froid prématuré avec d'importantes chutes de neige : le sentier, long d'environ 9 kilometres et servant de plateforme à la voie de 0,50 qui relie le lac avec la station supérieure du câble de ravitaillement, devint rapidement impraticable mêne aux mulets, et le ravitaillement dut être fait à dos d'homme. Le froir atteignit $-14^{\circ}$.

Le débit de la galerie se maintint sensiblement constant pendant une période assez longue, pour autant du moins que l'on en pouvait juger d'après celui du Sousoueou, émissaire normal du lac, car l'accès à la galerie était devenue très difficile à la suite des chutes de neige. Il faut d'ailleurs noter que la tem- de glaise ; cet écoulement s'accompagnait du bruit caracteristique des cascades dans les canaux souterrains.

Dans ces conditions, on décida immédiatement dagrandir la faille en faisant sauter la dalle avec des explosifs, de manicre a découvrir une partie du boyau emprunté par les eaux et i abaisser d'autant le plan d'eat. Ce qui fut fait, apres quoi le personnel put redescendre le lendemain.

Une expédition analogue fut organise une dizaine de jours plus tard et on constata que le niveau du lac s'était abaissé de $1 \mathrm{~m} .50$ environ (v. fig. 5b). Vers la percée, les eaux ne s'écoulaient plus dans une fissure, mais à travers des eboulis qui furent déblayés sur $0 \mathrm{~m} .80$ de hauteur environ dans la zone die passage de l'eau.

Une nouvelle expédition eut lieu le 10 avril : l'équipe envoyée au lac put pénétrer dans la galerie de percée par le puits; une forte charge d'explosifs, placée au fond de la galerie, eut des effets considérables. Le débit passa de $500 \mathrm{l} / \mathrm{s}$. environ à $5 \mathrm{~m}^{3}$; la faille avait été ćlargie et les dóblais qui lobstruaient entrainés dans la galerie (v. fig. $\bar{x}$.).

Le niveau du lac se fixa a 2 mitres environ au-fessus de la voûte de la galerie, délini par un seuil en place que les explosions successives n'avaient pu disjoindre.

La suppression définitive de ce seuil, commencée en 1926 (v. fig. 5 (d), dut être retardée; en effet, les travaux en cours dans la chambre de robinetterie avaient nécessité la fermeture de la vanne de garde décrite plus haul : et l'orifice n'était plus découvert. Après la misc en place définitive de la robinetterie, le lac put être vidé ; le dérasement du seuil fut alors exéculé sans aucune difficulté au début de l'hiver 1927-1928. 
Vous pensens que divers enseignements peuvent être retires de ces travaux.

En premier lieu, il semble toujours désirable d'opérer plusieurs percées successives pour diminuer la charge d'eau sur l'orifice, Les travaux sont grandement facilites quand ces galeries sont indépendantes, car l'évacuation des apports naturels du bassin versat du lac se fait par la dernière galerie percée sans que le travail soit gêné dlans celles où l'on travaille encore. Les galeries ont généralement des longueurs croissantes depuis la supérieure jusqu'a la percée définitive; feur multiplication n'engendre donc aucune perte de temps si l'on peut travailler simultanément aux diverses attaques. Quant au surcroit de dépenses qui résulte de l'exécution de percées auxiliaires, il n'est pas négligeable; toutefois, le gain de temps qui résulte presque toujours du fait que loperation, décomposée en béments dont chacun est facile, rie une pente asse\% forte; on peut ainsi achever dans de bonnes conditions la percée dans le cas où, du fait du débouché dans des éboulis, le débit reste relativement faible malgré la réussite de l'opération; en outre, les venues d'eau, souvent importantes quand la percée est proche, sont evacuées sans devenir gênantes.

Enfin, il est éminemment souhaitable d'être fixé aussi exactement que possible sur la nature alu terrain aux points où les diverses galeries dojent déboucher lans le lac; l'ecartement en profoncieur (ies faleries doit en effet être déterminé d'après les facilités probables qu'offrira le débouché de chacune d'entreelles. Il faut avant tout éviter de déboucher sous l'eau dans une zone défavorable; il y aura bien souvent un gain important de temps à rapprocher deux galeries pour pouvoir au besoin vides par siphonage une tranche d'eau et déboucher à sec dans une région
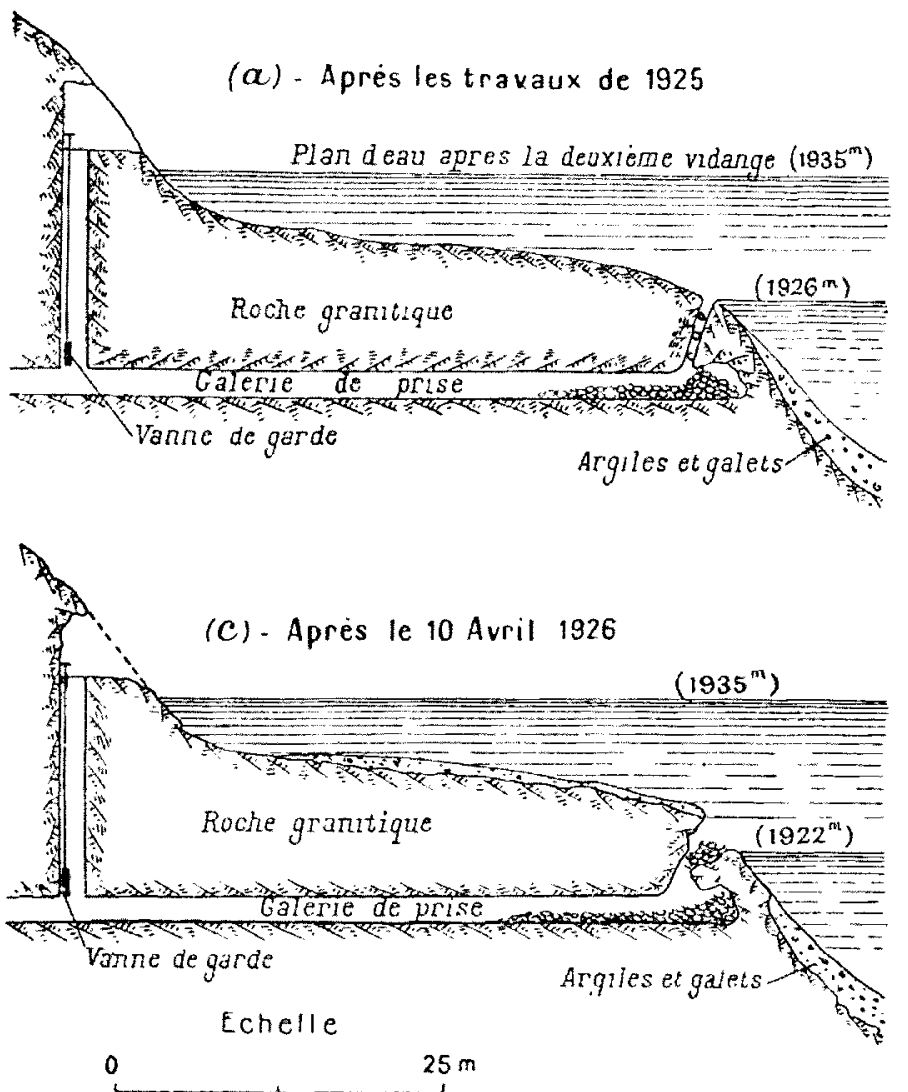

(b)- Apres les travaux de Mars 1925

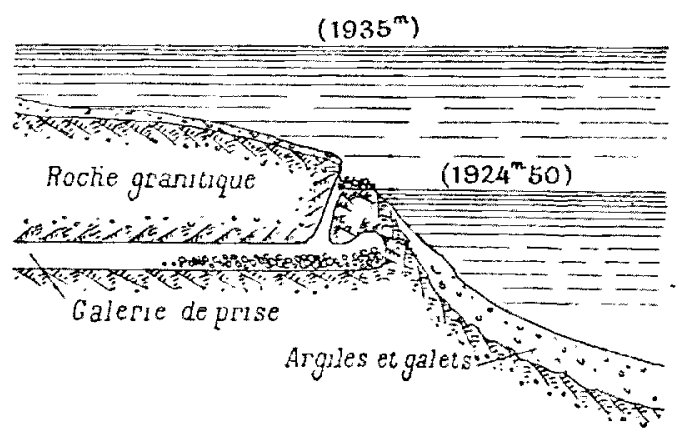

(d) Après la campagne dèté 1926

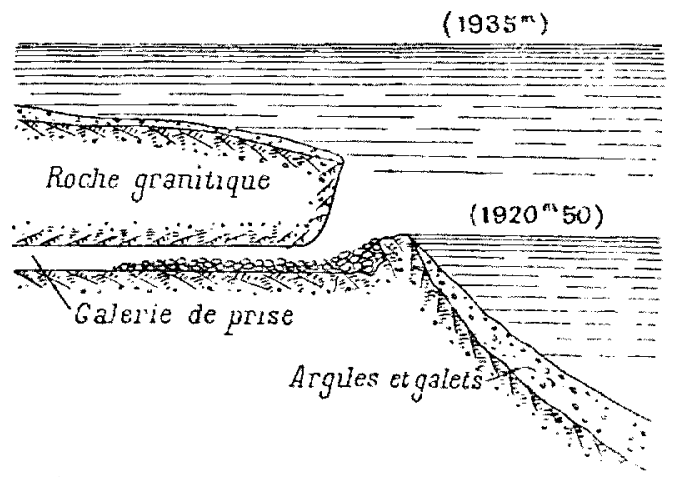

Fig. 5. -.. Iiverses phases de la cernière vidange du lac d'Artouste.

peut être conduite avec un imprévu minimum, compense et au delà le coût des galeries supplémentaires.

En second lieu, il est très avantageux de disposer chaque galerie de manière à pouvoir y circuler même si le débit qui y passe est important : il suffit de ménager une banquette ou d'approfondir le milieu du radier en caniveau, tout en donnant à la gale- dangereuse: au contraire un échec, outre ses conséquences morales fàcheuses, entraìne bien souvent, par le retard qui en résulte, un manque à gagner bien supérieur au surcroît de dépenses provoquées par une direction péchant par excès de prudence.

\section{Extrait des Annales des Ponts et Chaussees.}

\section{Développements hydro-électriques au Mexique}

De nombreuses études officielles en vue de travaux hydro-électriques ont été entrepris au Mexique, où l'on a constaté la possibilité d'exploiter jusqu'à 6.000 .000 CV. d'énergie hydraulique. Les installations actuelles ne dépassent pas $350.000 \mathrm{CV}$. Le plus gros producteur est en ce moment la Mexican Light and Power $\mathrm{C}^{\circ}$ Ltd, qui possède 5 stations centrales d'une capacité d'utilisation totale de $175.000 \mathrm{ch}$.

Cette compagnie cuvisage de consacrer 3 millions de livres anglaises dans ses travaux d'extensions. Lénergie produite est livrée à la ville de Mexico et aux mines d'argent de Pachuca par une ligne à $85.000 \mathrm{v}$; une autre ligne alimente les mines d'or et d'argent de El Oro et Angangueo. La mème compagnie possède encore huit petites stations hydro-électriques et quatre centrales thermiques. L'installation totale est evaluee à $200.000 \mathrm{CV}$. et produisit 561 millions de $\mathrm{kw} / \mathrm{h}$. en 1925.

La Orizaba and Light Power $C^{\circ}$ possède un réseau moderne qui alimente des régions éroignées telle que le port de Vera Cruz et Puebla, et fournit également l'énergie de traction aux chemins de fer électriques de montagne de la Compagnie Mexicaine des chemins de fer.

Ind. El. - - The Elctrical Review, 13 avril 1928.

\section{Les trains électriques à Manchester}

La ligne de chemin de fer entre Manchester et Altrincham doit ètre électrifiée et on dit que le travail doit être commencé ce mois-ci. La distribution se fera par ligne aérienne à une tension de 1.500 volts. On a proposé de construire deux nouvelles gares de voyageurs, l'une à Aston-on-Mersey et l'autre à Navigation Crossing. 


\section{Le programme d'électrification des chemins de fer allemands}

Il sagit de renseignements donnes lans une conference faite par M. Wechmann. Directeur des Chemins de Fer allemands. Leffectif des locomotives et automotrices scéleve à plus de 400 locomotives et 700 automotrices. La puissance globale peut ìtre éva-

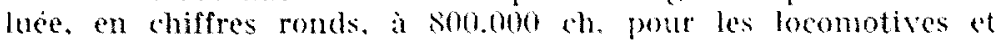
270.000 pour les automotrires.

libetrification don réseau de l'ampleur des chemins de fer du Reich demanderait des dizanes d'années, mème si tous les capitaux nécessaires étaient disponibles. Or, un programme s'étendant sur un pareil laps de temps ne saurait ètre immuable. Il est seulement prescrit dès aujourd hui que les constructions au-dessus de la voie doivent respecter le gabarit de passage des lignes catenaires et des trolleys. L'Allemagne a $2.500 \mathrm{~km}$. de ligne environ qui sont pratiquement prètes pour lélectrification. Ce sont naturellement celles dont la transformation réduira le plus possible les frais dex- ploitation par rapport a la traction a vapeur. Les Chemins de lier allemands utilisent pour la traction électrique sur les grandes lignes le courant altematif monophase a hasse frequence. Ce systeme est d'ailleurs adopté par la Suisse, l'Autriche la Suède et la Norvege.

L'office International des chemins de fer a fixé les normes pour la tension et la frequence, ainsi que pour les variations admissibles de ces valeurs. Tension nominale 15.000 volts, fréfuence nominale 16 23. Pour les lignes de ceinture et les lignes urbaines de Berlin, on a choisi le courant contimu a 800 volts. Le lype de courant pour certaines lignes d'intérêt local est choisi sclon les circonstances. par exemple. sur to ligne Berchtesgaden-konigsee, dans te Tyrol bavarois, le courant est du continu a 1.000 volts. M. Wechmann se declare tres satisfait du courant monophasé qui parait definitivement adopté pour les grandes lignes du Reich.

Girnic cinil, boctobre $192 \mathrm{~s}$.

\section{Mise en exploitation de la ligne de Bedous à}

Cette ligne prolongeant Pau-Oléron-Beslous, fut concédé à la Compagnic des Chemins de Fer du Midi, en même temp̧s que celle d'Ax-les-Thermes, à Puigcerda, en aoùt 1907.

Cest la première ligne perçant la chaine des Pyrénces. tont le trafic entre la France et l'Espagne se faisant actuellement par les deux lignes passant aux deux extrémités de la chaine.

L auteur expose les différents trajets envisages et donne le profil de celui qui a sté retenu, partant d'ume altitude de 106 mètres pour atteindre son point maximum au tumel du Somport a 1.211 mètres.

L'emploi de la traction électrique a permis d'avoir des rampes atteignant $43 \mathrm{~mm}$. par mètre, alors que la traction à vapeur ne peut permettre des déclivités supérieures à $33 \mathrm{~mm}$. Ceci a permis

\section{à Jaca, du chemin de fer transpyrénéen}

un trajet beaucoup plus court, ce qui est tris appreiciable daus un pay's où les travaux d’art sont si coûteux.

Nombreux détails sur les lravaux dart exécutés, en particulier pour la gare internationale de Canfranc.

Caracteristiques des locomotives electriques diun poids de 76 tonnes: vitesse maximum $75 \mathrm{~km}$ /heure: tension de la ligne 1.500 volts.

la ligne est alimentce par frois sous-stations : a bedous. aux Forges d'Abel et à Lrilos. Ces sous-stations sont alimentées par quatre centrales hydro-clectriques, produisant du courant i 6.0101 volts. $50 \mathrm{pps}$

Description de l'installation des sous-stations. le Ginie Cinil. 21 juillet 1928 .

\section{Joints d'huile pour les alternateurs refroidis à l'hydrogène}

On sait que le refroidissement, à l'hxdrogène, des alternateurs, d'abord utilisé pour les gyroscopes à commande électrique, permet d'accroitre la puissance nominale des machines de près de $30 \%$ tout en améliorant le rendement :

$1^{\circ}$ Empêcher la pénétration de l'air:

$2^{\circ}$ Réduire au minimum les fuites dhỵdrogène.

Dans ce but l'hydrogène placé à l'intérieur de la machine est à une pression un peu supérieure à celle de la pression atmosphérique. Mais, comme le manque d'étanchéité croit proportionnellement au carré de la surpression de l'hydrogene, il convient de prévoir des joints tries étanches, particulièrement au passage de l'arbre.

L’anteur représente en coupe. différents systèmes de joints ayant donné satisfaction. en mème temps les dispositifs auxquels il a eu à recourir pour faire des essais avec une surpression d'hydrogìne correspondant a une hauteur de colonne deau de $350^{\mathrm{m}}$. Certains des essais durèrent quatre jours.

Le facteur solubilite de throlrogene of de lair dans lhuile joue un ròle important.

Developpement mathématique des vitesses d'écoulement fors du démarrage et en marehe normale.

\section{Le grand électro-aimant de l'Académie des Sciences}

Les théories modernes de la physique exigent, pour leur recherche, des champs magnétiques intenses et de grandes surfaces : c'est, en particulier, le cas pour l'étude de la biréfringence magnétique.

Cne reduction au quart de lappareil prévu fut d'abord ríaliséc dans les ateliers de l'Office National de Recherches et Inventions et les différentes retouches et mises au point furent effectuces sur cet appareil d'essai.

L'étude et la construction de cet appareil, permirent de constater certains faits inconnus. Ainsi on reconnut que pour la prodnction du champ, la culasse n'a pas l'importance qu'on lui attribuait d'ordinaire.
L.appareil définitif fut construit par la Compagnie ThomsonHouston.

La culasse est constituéc par des poutres en acier: les noyaux sont en acier extra-doux forgé, de forme tronconique. Ils sont traversés par trois tubes concentriques destinés à manouvrer les pièces polaires. L'entrefer peut varier entre $0 \mathrm{~m} .750$ at $3 \mathrm{~m}$.

La puissance absorbée est de $100 \mathrm{kll}$.. sous 210 volts : nombre d'ampères/tour : 500.000 .

On a pu obtenir des champs de 16.1100 gauss entre des éprouvettes distantes rle $2 \mathrm{~cm}$.

Le Gónie Cioil, 28 juillet 1928

\section{La centrale hydro-électrique de}

Jusqu'ici la région industrielle de léningrad, dépensait uniquement pour l'énergie électrique les charbons importés, les charbon. nages du Donetz étant trop éloignés. Le projet d'utilisation du fleuve Wolchow remonte à l'époque d'avant-guerre, ce fleuve de $1.223 \mathrm{~km}$. de long sort du lac Ilmen et débouche dans le lac Ladoga. Le débit moyen est de $267,1 \mathrm{~m}^{3} /$ seconde et peut atteindre jusqu'à $1320 \mathrm{~m}^{3} /$ seconde. A $130 \mathrm{~km}$. de Leningrad ce fleuve forme des rapides pour une hauteur de $9 \mathrm{~m} .5$ sur une longueur de $10 \mathrm{~km}$.

Les travaux d'une centrale de $80.000 \mathrm{Ch}$. furent commencés fin 1921 et la centrale fut mise en service en 1927 .

L'auteur décrit les travaux ayant dû itre exécutés pour l'érec-

\section{Wolchowstroi, près de Léningrad}

tion du barrage réservoir mesurant 210 metres de long, $18 \mathrm{~m}$. de haut et $39 \mathrm{~m}$, de large.

La centrale comporte 8 turbines hydro-électricjue de 10.000 ch. Turbines Francis à une roue, à arbre vertical, tournant à $75 \mathrm{t.} / \mathrm{ml}$. ; rendement $86 \%$.

I.es alternateurs ont un rendement de $85 \%$ et un cos. $\varphi$ de 0.8 . Ils débitent sous 11.000 volts à 50 pps.

Coupes de ces machines, de la centrale; caractéristiques des transformateurs, des disjoncteurs et de la ligne de $\$ 30 \mathrm{~km}$. alimentant Léningrad, sous 5.000 volts.

E. T. Z., 2 aotit 1928. 


\section{Lumiére et visibilité}

Les qualitio que doit présenter un Jon éclairage et les avantages économiques résultant de l'éclatrage rationnel d'un atelier sont connus.

Mais f'influcence de l'effel des vibrations sur la visibilite est tune question intiressante qui proccupe les techniciens chargés de l'éclairage des vehicules en mouvement.

M. W. J, Jones rappelle des essais effectues sur les chemins de fer de 13akerloo, avec un éclairement de 20 lux sur un texte de journal imprimé aver des caractères de 8 points.
La distance a laquelle il etait possibje de lire exactement, quj était de $0 \mathrm{~m}$. 90 au repos, était de $0 \mathrm{~m}$. 75 pendant la marche et la lecture s'en trouvait gènée. Ljeffet des vibrations réduisait la visibilité de $33 \%, 140$ mots par minute au lieu de 200 mots.

Jes résultats sensiblement équivalents ont été effectués sur des plate-formes de tramways et sont signalés par l'auteur.

Les valeurs des eclairements admises pour rles installations fixes doivent donc ètre dépassées pour des véhicules.

The Illuminating Engineer, septembre 1928.

\section{Appareil d'électrolyse produisant directement l'hydrogène et l'oxygène à haute pression}

La fabrication directe de l'hỵlrogène el de l'oxygène à haute pression par l'électrolyse de l'eau, présente de grands avantages. Mais la réalisation des appareils nécessaires à cette fabrication soulève des difficultés de construetion, sous le rapport de la solidité, de la durće de l'étanchéité et aussi de l'isolement. quand on monte les éloments en série. I'n ingénicur allemand, $\mathrm{X}$-.M. Moegnolrath, a réalise un électrilyseur. fonctiommant à 150 atmosphères, de dimensions relativement restreintes.

Les élements ćlectrolyseurs à haute pression sont constitués par des tubes dans l'axe desquels sont disposées les cathodes en nickel. Elles sont entources par des parois, non représentíes, qui les sépa- rent des électrodes positives, l'électrolyse étant de la potasse. Ces éléments électrolyseurs sont montés en série dans un ordre bien déterminé. Les essais effectués ont montri que le nouvel électrolyseur consomme 3 à $3,5 \mathrm{kw}$ par mètre cube de gaz.

La pureté atteignit 99,8 pour l'oxygène et 99,9 pour l'hydrogène. L'électrolvseur sous pression présenterait les avantages suivants :

Ilaut rendement pour les densités de courant élevées la consommation est de 3 à $3.5 \mathrm{KW}$ h. à 200 atmosphères; grande pureté des gaz: faible encombrement: possibilité d'installation à l'air libre; diminution des frais de surveillance; absence de risques d'explosions.
Génie Civil, 18 août 1928 .

\section{Calcul économique des lignes de transmission d'énergie électrique}

Dans le cas ou ha tension n'est pas fixée a l'avance par une raison pratique quelconque, les calculs de lignes sont ordinairement faits par tatomements. Cne méthode de calcul direct unique a cté etablie en 1925 par $F$. Kirsten ot E.-A. L.ow ; elle fait intervenir les quatre domnées suivantes : 1" valeur totale des dépenses correspondant aux pertes en ligne: $2^{\circ}$ charge ammuelle correspondant a lamortissement du prix dachat des conducteurs: 30 charge anmuelle correspondant à la partic du prix des supports qui varie avec la section des conducteurs: to charge annuelle relative au prix des appareils de ligne dans la mesure où celui-ci varie avec la section des càbles. les formules clonnant ces diverses charges pouvant toutes s'exprimer en fonction de la section des conducteurs et de la tension, il est possible de les combiner pour arriver à une formule unique dans laquelle n'entrent que les constantes habituelles des calculs de ligne et trois coefficients qui expriment respective- ment : a) le rapport entre le diametre du conducteur et la tension de ligne, établi d'après la loi de Peek sur l'effet de couronne: b) le coùt des pylònes en fonction de la tension: c) le coût des appareits de ligne par unité de longueur de cette dernière. L'auteur donne dans l'article la raleur de ces constantes et coefficients pour permettre d'étendre la méthorle exposée à tous les types de lignes de transmission actuellement en usage aux Etats-linis. Il termine en reproduisant une série de courbes au moyen desquelles les valeurs de la tension et de la section des câbles d'une ligne peuvent être déterminées par simple lecture d'après la longueur de la ligne et la puissance à transmettre. Un exemple concret appliqué a une ligne double de $160 \mathrm{~km}$ de longueur et transmettant 80.000 $\mathrm{kv}$-A montre comment ces différentes courbes doivent ètre utilisées. - 13. E.

\section{Interprétation de nouvelles expériences effectuées avec le klydonographe sur le fonctionnement des parafoudres de lignes}

lauleur interprete un grand nombre de relevés faits au moven du klyonographe sur de's ligne's aériemnes pourvues des parafoudres. Les experiences ont d'abord montré que la zone pour laquelle des décharges atmosphériques produisent sur la ligne des surtensions dangereuses se limite à une étroite bande de $400 \mathrm{~m}$. de largenr environ de chaque còté de la ligne. D'autre part. les observations métcorologiques indiquent, pour les états centraux des Etats-Unis, une moyenne de 50 journées d'orage au maximum, par an. bans ces conditions le nombre de décharges dangereuses par an, en chacule point, serait seulement de 5 à 10 . D'autre part, lorsqu'on considère cue les parafoudres doivent ètre réglés pour 3.5 fois la tension de la ligne. les deux tiers des décharges seulement produisent un arc. Cependant, par suite des caractéristiques actuelles et de l'énergie relativement faible qui est transmise apres production d'un arc au parafoudre, la tension est réduite à une valeur qui n'est plus dangereuse après un parcours assez faible. Toute tendance à angmenter l'isolement de la ligne accroit cette distance et augmente donc la nécessité d'utiliser des parafoudres. L'auteur signale que, dans plusieurs cas, et pour des lignes de tension inférieure à $33 \mathrm{kv}$, l'installation de parafoudres a réduit d'environ 80 pour 100 à 95 pour 100 le nombre d'accidents dus aux décharges atmosphériques et il conclut en disant que ces appareils se révèlent aussi utiles sur les lignes à basse tension que sur celles à très haute tension. Les essais ont d'ailleurs démontré qu'il n'était pas possible de se rendre compte de la valeur des parafoudres par des expériences de laboratoire à cause de la quantité d'énergie limitée dont on dispose. L'auteur termine en constatant que le klvdonographe est un appareil pré. cieux, donnant de très utiles indications sur le problème étudic. mais qu'il ne permet pas à lui seul de le résoudre: il semble que l'oscillographe à rayons cathodiques conviendrait bien pour ce genre de recherches. $\quad$ B. E. J. A. I. E. E., août 1928.

\section{Les redresseurs au tantale}

Après quelques considérations genérales sur les conditions de recharge des accumulateurs et sur les redresseurs électrolytiques, l'auteur traite des redresseurs où les deux dectrodes sont unc lame de plomb et une lame de tantale plongeant dans un électrolyte compose diacide sulfurique a $22^{\circ} \mathrm{I}$ is contenant $20 \mathrm{gr}$. de sulfate ferreux par litre. Cette soupape est traversée par le courant quand le tantale est négatif par rapport au plomb; le courant est arrêté dans le cas contraire. Dans le moddle étudié par l'auteur la lame de tantale avait $7 \mathrm{~cm} \times 1.7 \mathrm{~mm}$ of $0,1 \mathrm{~mm}$ d'épaisseur : l'autre électrode était notablement plus large et plus épaisse. Pour des tensions supérieures i $3 \mathrm{v}$, le débit de l'appareil croit rapidement avec la surface immergéc des électrodes; par contre il varie très peu lorsqu'on rapproche celles-ci (le courant passe de 0.8 à $0,84 \mathrm{~A}$ lorsque l'écartement diminue de 10 à $4 \mathrm{~mm}$ ). La tension critique de ce redresseur est. en courant alternatif, de $38 \%$ ce qui donne environ 35 v pour la tension maximum a laquelle peut fonctionner un élément : on est donc conduit à employer pour la charge d'une batterie de $80 \mathrm{v}$ sur un courant alternatif à $110 \mathrm{v}$ au moins 4 éléments en série. Contrairement à ce qui se passe dans les redresseurs dont l'électrode active est une lame d'aluminium, l'échauffement de l'électrolyte n'a pas une grande influence sur le fonctionnement de ce redresseur.- - B. E.
La T. S. F. moderne 


\section{De la théorie des courbes d'échauffement et de refroidissement de machines et d'appareils électriques}

Pour élablir les formules dissiques qui domnent bechatfement et le refroidissement des machines et des appareils dectriques an fonction du temps, on admet que la production de chaleur. le coefficient de transmission de cette chaleur, la température ambiante ou du milieu réfrigérant ef celle d'un point à un autre du corps considéré. restent imvariables, ce qui n'est pratiquement pas le cas. Ordinairement la production de chaleur augmente avec la température de la machine malgré la constance du courant de charge et, dans bien des cas, le coefficient de transmission de la chaleur croit aussi avec l'échauffement. Il convient en outre de faire une distinction entre les machines a refroidissement clirect et celles à refroidissement indirect par un réfrigérant intermédiatre. Les premières cèdent leur chaleur à un milieu réfrigérant illimité dont la température peut ètre admise comme constante. Dans les secondes intervient le réfrigérant intermédiaire dont la température est variable ce qui dome lieu a deux échauffements, des refroidissements partiels exercant l'un sur l'autre une action mutuelle qui complique le problème. Cette action est négligeable quand la constante de temps de la machine est assez faible par rapport à celle du réfrigérant internédiaire, comme par exemple dans les transformateurs à hain d'huile, mais d'autres. complications surgissent lorsque la résistance des conducteurs de courant augmente avec la température. Le dégagement de chaleur d'une machine se produit par convedion at par rayomement. Son refroidissement est naturel ou artilicicl suivant que l'agent refrigérant est immobile ou en mouvenent par rapport à la surface de la machine. Alors que, dans le second cas, le coefficient de convection est constant, il augmente dans le premier cas avec l'chauffement de la machine: le degagement de chaleur par convection naturelle est proportionnel à la puissanee 1,25 de la température d'échaulfement. Quant au dégagement de chaleur par rayonnement il peut aussi etre admis proportionnel a cette mème puissance 1,25 de la température d'ćchauffement entre $20^{\circ}$ et $100^{\circ}$ C environ. Sur la hase de ces considérations, l'auteur traite le probleme mathímatiquement pour le cas du refroidissement artificiel et cehi du refroidissement maturel. Dans le premier cas il constate que l'expression qui correspond is la constante de temps de la théoric classique n'est pas une (onstante absolue, mais une fonction de léchauffement final, cest-a-tlire de la charge, avec laquelle elle augmente, de sorte que léchauffement est plus lent et le refroidissement plus rapide que no l'indique la theorie classique. Dans le second cas, cette expression ne dépend pas seulement de l'échauffement fimal. done de la charge. mais encore de l'échaufement à chacue instant, cest-i-dire du temps. Un cas particulier est celui du refroidissement l'une machine hors circuit, par l'examen ducpuel l'auteur termine son exposé. C. A. E. T. Z., 2 aout 1928.

\section{Les grands réseaux de chemins de fer français de 1878 à 1928}

Cette étude d'ensemble, parue à loccasion du cinquantième anniversaire de la fondation de la "Revue générale des Chemins de fer $n$, se subdivise en plusieurs chapitres. L auteur traite d'abord de la situation des grands réseaux de chemins de fer francais en 1878, au point de vue de leur étendue. de leur régime légal et financier, et de leur situation technique (exploitation, materiel roulant, traction et voie). Le second chapitre est consacré au développement des réseaux jusqu'i la guerre de 1914: on y trouve des renseignements sur le rachat du réseau de l'Ouest, sur les progrès techniques et sur la situation matérielle et morale à la veille de la guerre. Ja guerre et les crises corrélatives sont étudiées dans un troisième chapitre qui traite de l'effort des chemins de fer pentlant la guerre 191 $1-1918$, de la crise matćriolle des transports pendant cette période (surmenage et usure du matériel, destructions, effort de redressement) et enfin de la crise morale (loi de huit heures, grèves de 1920 et nouveau statut du persomnel). Dans le quatrième chapitre, des indications sont données sur le régime actuel des grands réseaux français de chemins cle fer d'intérèt général et sur leur régime antérieur (conventions de 1883 et de 1921). Le cinquième chapitre, de beaucoup le plus important, examine en détail la renaissance et le développenent des chemins de fer français depuis les crises de 1919 et 1920 jusqu à 1928: on y trouve des renseignements sur les progrès technicues réalisés pour lexploitation (circulation et sécurité, installation des gares), le matériel et la traction (dépôts et ateliers, voie, matériel roulant, nouveaux types de locomotives à vapeur, electrification); ce chapitre se termine par l'étude des mithodes commerciales des grands réseaux de chemins de fer et des progrès réalises dans ce lomaine. Un tableau complémentaire domne pour les années $1878,1888,1898$. $1908,1913,1919$ et 1927 les chiffres totaux concernant les résultats d'exploitation (receltes, dépenses, produit net et coefficient d'exploitation). ceux concernant le service des voyageurs, des marchandises petite vitesse et enfin leffectif et les parcours du materiel roulant. Cette etudo d'cnsemble peut se resumer brievement par la comparaison des chiffes suivants : a) l'itendue moyeme exploitée est passée de $19.841 \mathrm{~km}$ on 1878 a $39.754 \mathrm{~km}$ en 1927, tandis que le capital net inverti (actions el obligations) qui était de 8 milliards de francs en 1878 sest élevé à près de 4t milliards aujourd'hui : b) le matériel roulant est passé, dans la mème période, de 6.516 locomotives à 19.272 . de 1.1 .787 voitures a $31.7+7$ et de 172.983 wagons et fourgons à $199.606 ; c$ ) au point de vue régime, les conventions successives de 1859.1883 et 1921 ont consacré un accroissentent constant des pouvoirs de l'Ftat et (le son intervention dans le partage des profits; d) en matière de perfectionnements techniques, les traits dominants sont l'accroissement de la sécurité et du débit de la circulation. ainsi que le souci d'une économie plus grande de charbon, d'où dérivent en particulier les programmes d'électrification. - - F. P.

Revue génirale des Chemins de fer, juillet 1928.

\section{Les chemins de fer français d'intérêt local de 1878 à 1928}

Cette courte étude, parue à l'occasion du cinquantière anniversaire de la fondation de la "Revue générale des Chemins de fer " traite du développement des chemins de fer d'intérêt local en France au cours des cinquante dernières années. File comporte l'examen des tendances des exploitants de ces réseaux sous les régimes successifs des loís du 12 juillet 1865 , du 11 juin 1880 et du 31 juillet 1913 ; à l'époque de cette dernière loi, Je réseau des chemins de fer d'intérêt local était cinc fois plus étendu qu'en 1878 et comportait $22.000 \mathrm{~km}$ de lignes concédées dont $17.500 \mathrm{~km}$ en exploitation et $4.500 \mathrm{~km}$ en construction ou en projet; il comptait 2.079 locomotives, 5.118 voitures de 36 places en moyenne, 1.253 fourgons et 19.975 wagons d'une capacité moyenne de 9 t.; le personnel se composait dc 32.300 agents pour un trafic (1912) de 85 millions de voyageurs et de 15 millions de tonnes de marchandises. Le bouleversement économique de la guerre a affecté tout particulièrement l'exploitation dies lignes d'intérèt local et a conduit à plus de 600 décrets de relevenents de tarifs pour la période de 1916 à 1919 . En raison de la concurrence des transports automobiles, qui sont souvent plus économiques que ceux par voie ferrée, les lignes d'intérèt local ont dù cyoluer dans le sens de la simplification et de la réduction des depenses, pour pouvoir soutenir avec succès la lutte dans le domaine qui leur senble réservé du transport des marchandises lourdes et en grosses quantités industrielles. F. P. Revue générale des Chemins de /er, 7-1928.

\section{Les chemins de fer des colonies, protectorats et territoires sous mandat}

Cette étude, parue à l'occasion du cincquantième anniversaire de la fondation de la "Revue générale des Chemins de fer ", est consacrée au développement des voies ferrées dans les colonies franf̧aises. Elle est subdivisée en plusieurs parties. Ia premiere traite de la situation pour l'Algérie, la Tunisie et le Maroc ; la seconde est consacrée aux colonies autres que l'Algérie (Indo-Chine, Afri- que occidentale, Afrique équatoriale, Madagascar et colonies diverses); la troisième partie enfin expose les résultats obtenus dans les territoires sous mandat (Syrie, Cameroun, 'Togo). Pour chacun des pays sont domés également les projets en cours d'étude. F. 1 .

Revue générale des Chemins de fer, juilld 1928. 
Le calcul simplifié par les procédés mécaniques et graphiques, par M. D'OCAGNE, membre de l'Institut, professeur a l'Ecole polytechnicue. In volume in-8 de 206 pages avec 69 figures. Troisieme intion, aver une rérlaction entièrenent renonvelé et de nombreuses additions. Gauthier-Villard \& Cie, editeurs. En vente at la librairic Arthaud-Rey, Grande-Rue, (irenoble. Prix : $20 \mathrm{lr}$.

En offrant à l'Acadénie la troisième édition qui vient de paraitre, de son livre intitule "Le calcul simplifié par les procédés mécaniques et graphiques ", M. l'ocagne dit que, tant par le profond remaniement des parties conserves de la précédente édition que par les nombrenses additions introduites dans la nonvelle, celle-ci constitue, à proprement parler, un Ouvrage nouveau dommant une vue d'ensemble, exactement mise au point, de tous les procédés d'une extrême varictí qui ont ćté tirés de la géométrie et de la mécanique, en vue de suppléer au calcul numériçue pour les besoins des diverses branches de la science et de la technique.

Dans cette nouvelle édition, l'Ouvage est divisé en cind chapitres correspondant aux cing classes définies dans la communication faite par l'auteur à l'Acalćmie le 18 janvier 1926, en lesquelles il est parvenu à ranger tous les procédés en question :

Calcul numérique (instruments et machines arithmétiques); Calcul graphique (algère, statique et intégration graphicues) Calcal grapho-mécanique (intégrometres et intégraphes): Calcul nomogruphique (abaques et monogrammes) ; Calcul nomomécanique (instruments et machines logarithmiques).

Lohjet de l'Ouvrage peut etre ainsi résumé : dire en quoi consistent tous ces procédés; exposer succinctement les principes genéraux sur lesquels iss reposent : faire comnaitre le genre diutilisation auquel peut plus particulièrement se prêter chacun d'eux; fournir enfin, en vue d'une ctude plus approfondie de tel ou tel d'entre cux, des indications bihliographiques aussi completes et précises que possible.

(S. Y.).

La Technique du bobinage des machines électriques (dynamos, alternateurs, moteurs, transformateurs), par BARdrs, ingénieur électricien. Ln volume in-16 ke 72 pages. 36 figures. Desforges, Girardot \& cie, éditeur. En vente à la librairie Arthaud-Rey. Prix : 6 fr. 50.

Cet ouvrage étudie les differents modes denroulements utiliscis dans les machines électriques. en développant les modes de construction employes dans les divers systemes inducteurs et induits.

Un avant-propos rappelle les propriétés des courants alternatifs simples et polyphases, ainsi que les propriétís du courant continu. Cet avant-propos se borne aux comaissances qu'il est indispensable de connaitre, pour hien comprendre la technique des enroulements.

I.'auteur examine ensuite, en détail, les enroulements utilisés dans les inducteurs et induits des dynamos à courant continu, des alternateurs monophasés et polyphasés, des moteurs à courant alternatif et des transformateurs. Chacune de ces itudes est prisentée sans mise en ceuvre de formules compliquées et complétée par une partie pratique sur la confection de ces divers enroulements.

En resumi, le technicien ef le praticien trouveront, dans cet ouvrage, les béments nécessaires pour la confection ou la réparation des enroulements des machines dectriques; comaissances condensées sous la forme la plus simple possible, demandant le minimum de temps pour s'initicr ou se perfectionner sur cette fuestion importante, que tous les électriciens ou usagers de machines ilectriques doivent connaitre.

(S. V.).

\section{$\therefore$}

L'Amérique économique, par le I ${ }^{r}$ Carl KoETrGen, directeur général des usines Siemens-Schuckert, vice-président de l'office allemand de recherches économicues. Un volume in-s de 200 pages avec 12 gravures dans le texte et 28 illustrations hors texte. Bibliotheque politique et économique. Payot, 106, boulevard Saint-Germain, Paris, éliteur. En vente à la librairje ArthaudRey, Grande-Rue, Grenoble. Pris : $25 \mathrm{fr}$.

Un livre qu'on ne se contentera pas de lire mais que l'industriel, le commerçant, garderont sur leur table de travail pour en con- sulter fréquemment les tableaux et les graphiques extraordinaires de netteté.

L'Europe d'après guerre envie la grande prospérité américaine ct voudrait savoir le secret de cette puissance agricole, commerciale, industrielle et financiere.

Les grands maîtres des affaires américaines nous ont exposé leurs méthodes. Ces méthodes sont-elles applicables à l'Europe ? C'est ce qu'a voulu itudier M. Koettgen, directeur général des usines Siemens-Schuckert, une des plus grosses firmes industrielles allemandes, célèbre dans le monde entier. Il a rapporté d'un long voyage technique aux Etats-Unis ce volume plein de chiffres, de tableaux et de graphiques qui. pour la première fois, donne des renseignements numériques exacts, précis et compétents sur l'activité américajne. La conclusion de ce précieux travail -- conclusion un peu inattendue de la part d'un grand industriel -..- c'est que la base de la prospérité des Etats-Lnis est l'agriculture. C"est l'intérêt tout nouveau de cet ouvrage fondamental qui renouvellera bien (les idées en France sur un sujet rapital : les Ftats-Unis.

(S. V.).

\section{$\star$}

Théorie industrielle de l'électricité et des machines électriques. Par A. Vendcrand, ancien élève de l'Fcole Polytechnique, préface du Général Ferrié. - Un volume $16,5 \times 25^{\mathrm{c}}$, de 483 pages, 342 figures dans le texte. - Librairie $H$. Dunod, éditeur. En vente à la librairie Arthaud-Rey, Grande-Rue, Grenoble. Prix broché : 63 francs.

Comme le dit le général Ferrié dans sa préface, l'ouvrage du capitaine Verdurand est établi suivant un plan très original, qui le différencie très nettement des traités d'électricité antérieurs. Dans la partie théorique, exposée surtout par le raisonnement, il est fait appel au bon sens du lecteur plutôt qu'à ses connaissances mathématiques.

L'auteur considère la machine polyphasée comme la plus simple et en déduit la dynamo à collecteur. D'autre part, il représente le transformateur triphasé à champ tournant comme la machine type à champ tournant, de même que le transformateur statique monophasé sert d'introducteur aux machines à champ alternatif.

Cet important ouvrage est au courant des nouveantés de l'électrotechnique et contient des renseignements très complets et exposés avec une grande clarté, sur le compoundage des alternateurs les groupes convertisseurs, les montages en cascade de moteur asynchrone et de machine à collecteur pour la commande des laminoirs, la régulation des moteurs, détraction, etc.

Nous ne saurions trop reconmander cet ouvrage aux Elèves Ingénieurs de nos grandes écoles, et à toutes les personnes qui déjà dans l'industrie, ont besoin d'être au courant du fonctionnement des machines électriques.

\section{Electrochimie appliquée. Electrolyse de l'eau et des chlorures} alcalins, par Jean Billiter, professeur à l'Université de Vienne. Traduit d’après le 2 édition allemande par J. et $S$. Salauze, ingénieurs chimistes. --.. Volume $16 \times 2.5, X-135$ pages, 262 figures $(810$ gr.). Relić, $94 \mathrm{fr}$. Brochc, 81 fr. Dunod, editeur, 92, rue Bonaparte. Paris $\left(6^{\mathrm{e}}\right)$. En vente à la librairie Arthaud-Rey, Grande-Rue, Grenoble.

Depuis quelques années déjà, ggràce à son magnifique développement, l'électrochimie est devenue une branche fort importante de l'industrie chimique. L'ourrage du Professeur Billiter fournit aux Ingénieurs, aux Industriels, aux Chimistes, un exposé clair, méthodique et précis des urocédés mis en cuvre dans les diffé rentes branches de ce que l'on peut nommer "la grande industrie électrochimique ".

L'ourage débute par une étude des phénomènes de surtension qui sert de guide. Puis sont exposés les différents procédés industriels mis ell ceuvre pour l'électrolyse de l'eau. Deux chapitres sont consacrés aux réductions et axydations des composés minéraux et organiques. Vient ensuite un exposé complet de l'électrolyse des chlorures alcalins, de l'obtention du chlore et des débouchés de ce gaz : dérivés chlorés organiques et chlorure de chauxdu traitement des lessives alcalines, de la fabrication electrolyti, que, des hypochlorites des chlorates et des perchlorates. Les mé- 
thodes damalyse des produits obtenus et de contrôle des fabricitions sont exposees et discuties. Enfin l'ourage se termine par quelques pages sur l'blectrolyse des solutions de bromure et sur les réactions électrolytiques d'additions et de substitutions d'halogenes. Il constitue une source de documentation absolument remarquable.

(S. V.).

Le chef mécảnicien et électricien, par E. BLANc, ingénieur électromicanicien. Tome IV. Un volume ill-16 broche, de 607 pages, avec 230 figures. Desforges, Girardot \& C céditeurs. En vente à la librairie Arthaud-Rey, Grande-Rue, Grenoble. Prix : $47 \mathrm{fr}$.

Ce quatrième volume de la collection "Le chef mécanicien électricien " traite l'électricité générale, et présente la même clarté d'exposition que les précédents.

Après l'itude des phenomènes et de's lois de lélectricité, l'auteur átudie les mesures, les piles et les transformateurs, puis les dymamos et alternateurs, pour arriver ensuite ì lutilisation des courants : eclairage, distribution et télecommunication. Ce traité élémentaire, necessaire à celui cui veut ćtudier seul l'ćlectricité, rendra de grands services aux monteurs, aux contremaitres. aux hlèves des Ecoles professionnelles, et aura certainement le même succès que les autres ouvrages de cette collection.

(S. V.).

\section{$\therefore$}

Coup d'œil sur la production de l'énergie électrique en France, par MM. E. Ricibr et M. Laborde. Tne brochure, 103 pages, 6 tableaux, une carte. Aoùt 1928. Edition de la Remue Industrielle, 3, rue de Messine, Paris ( $\left.{ }^{\mathrm{e}}\right)$. Prix : 6 francs. Franco :

9 francs. - En vente à la librairie Arthaud-Rey, Grenoble.

Pour justifier son titre, cet ouvrage apporte sur la procluction de l'énergie électrique en France, des indications aussi actuelles que possible en même temps que très schématiques. Il comprend deux parties :

$1^{o}$ Ln inventaire de la situalion actuelle, consistant surtout en une étude statistique, basée sur les renseignements officiels. L'intérêt particulier de cette étude résulte non seulement dle l'ntilisation des documents les plus récents, et en particulier de la dernière statistique du Ministère des Travaux publics (actuellement en cours d'impression) mais encore d'une présentation originale qui a permis d'obtenir des indications trés précises sur l'évolution des situations régionales.

Les résultats sont conderssés en trois tableaux dont une lecture attentive permet de dégager toutes les caractéristiques de la situation actuelle.

Les auteurs ont complété l'inventaire précédent par une analyse des conditions probables de développement de la production d'énergie électrique dans l'avenir immédiat et ils ont chiffé leurs prévisions pour la période de dix ans qui va de 1927 à 1936. Ces prévisions font lobjet des quatrième et cincuième tableaux.

$2^{\circ}$ Un examen pour chaque région des projets actuellement en cours d'exécution ou sur le point d'être entrepris.

Alors que l'inventaire précédent a un caractère de certitude, en raison des éléments officiels qui ont servi à l'établir, les résultats de cette deuxième partie du travail ne peuvent être qu'approximatifs, tant par l'incertitude des dates et des conditions de réalisation, que parce qu'une partie des projets a pu échapper aux investigations des auteurs. Malgré ces difficultés, le bilan général qu'ils présentent dans leur dernier tableau est des plus significatifs.

La détermination des besoins nouveaux prévisibles d'ici à 1936 et l'évaluation des ressources nouvelles que l'on a décidé de créer au cours de la même période conduisent à un certain nombre de conclusions. Malgré leur désir de laisser chaque lecteur les dégager pour son propre compte, les auteurs n'ont pas pu en éluder un bref examen, examen qu'ils se proposent de développer ultérieurement.

La partie constructive de ce travail -... prévisions et conclusions - sera peut-être discutée. Sa partie documentaire puisée aux sources des meilleures et les plus récentes, s'imposera comme l'un des ensembles les plus complets et les plus équilibrés qui aient été présentés sur la question traitée.

Une bibliographic abondante el en grande partie originale complète très heureusement cet ouvrage.
Les grands barrages en maçonnerie aux Etats-Unis. - 1'ar M. I) I:sove, inginieur des Ponts et Chanssées. In volume $21.5 \times 32$ de 90 pages. XXIl planches hors texte. -.. Librairie de l'Enseignement technicue lion liyrolles, éditeur. bin vente a la libriric Arthaud-Rey, (irande-Rue, (irenoble. Prix : 35 franes.

limportant ouvrage de $M$. Jegove est le résultal d'un voyage d'étude cu'il a effectue aux Etats-Unis, pays of frant une collection très importante de grands harrages, étudies et construits recomment, suivant les méthodes différentes de colles qui ont iti employées en liranee.

M. Degove a recueilli des indications abondantes sur les projets aupres des ingénieurs du Reclamation Service, flu Service des Eaux de New-York, San-lirancisco, Denver, Los Angeles, San-Jiego, et plusieurs Indénieurs-Conseils qui se sont occupés de grancis barrages, le tout complété par la lecture de nombreuses publications amćricaines.

Dans ce mómoire relatif aux grands barrages en maçonncrie, l'auteur a abordé la plupart des problèmes que peuvent se poser à rette orcasion, les ingénieurs et les Fntreprencurs, sans toutefois avoir la prétention de les résoudre, mais avec l'éspoir de suggérer des solutions par la connaissance de celles qui ont été appliquécs avec succes aux Etats-Unis.

Louvrage contient de nombreux tableaux, heaucoup the photographies et de plans qui aident le lecteur a se représenter plus nettement les mincipaux barrages et chantiers de construction décrits et de découvirir une foule de détails pratiques intéressants que le texte ne fournit pas.

\section{$\stackrel{*}{*}$}

(S.V.).

Transport de l'énergie électrique. -..- l'ar H. Kysls, ingénieur, tratraduit sur la deuxieme edition allemande par R. Wesker et D.rvid. - 3 volumes de 130,138 et 1.008 pages. Librairie polytechnicpue Béranger, ciliteur. In vente at la librairic Arthaud-key, Grande-Rue, Grenoble. P'rix reliés 90, 91 et 182 francs.

Il sagit d'un travail tres important sur la production, le transport et l'utilisation de l'énergie électrique, faisant l'objet de 3 tomes.

Le Tome I traite rles moteurs, des convertisseurs, des transformateurs, dẹ leur fonctionnement, de leurs couplages, de leurs applications et de leur construction.

L.e Tome II traite des canalisations à basse et haute tension, de l'étahlissement des projets, calculs, avec de nombreux renseignements sur les dispositions électriques et mécaniques.

Le fome Ill, très important, concerne les usines génératrices machines à vapeur, chaudieres, combustibles et foyers. Locomotives à vapeur, moteurs Dirsel, moteurs à gaz, turbines hydrauliques emploi des batteries d'accumulateurs, appareillage électricue. Exploitation des usines genératrices. Etude des projets au point de vue ceonomique.

Ces onvrages, de par lcur importance, trouveront leur place dans toutes les bibliothèques des Ingéuieurs.

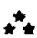

Ce que doit savoir tout Administrateur de Société Anonyme, par A. Moreny, rélacteur au Journal des Notaires. - Un volume de 120 pages, format $16,5 \times 25,5$. - - Administration du Recueil général des lois, éditeur. - En vente à la librairie Arthaud-Rey, Grenoble. - Prix : 12 francs.

L'Administrateur d'une Société anonyme endosse une double responsabilité : responsabilité civile, responsabilité pénale. Il devrait donc, en principe, comnaìtre très exactement la nature et l'étendue du mandat qui lui est confié, ses pouvoirs, ses droits et ses obligations depuis l'époque de la constitution de la Société, jusqu'à sa dissolution ou sa licjuidation.

L'ouvrage de M. Moreau est une étude tries complete et d'une consultation facile, des pouvoirs, des droits, et des responsabilites de l'Administrateur d'une Société anonyme.

(S. V.)

Le Gerant: J. Bataillann. 